\title{
Cavitation Bubbles Mediated Molecular Delivery During Sonoporation"
}

\author{
Tetsuya KODAMA ${ }^{* *}$, Yukio TOMITA ${ }^{* * *}$, Yukiko WATANABE ${ }^{* *}$, \\ Kenichiro KOSHIYAMA ${ }^{* * * *}$, Takeru YANO ${ }^{* * * * *}$ and Shigeo FUJIKAWA ${ }^{* * * * * *}$ \\ ${ }^{* *}$ Graduate School of Biomedical Engineering, Tohoku University, Japan, \\ 2-1 Seiryo, Aoba, Sendai 980-8575, Japan \\ E-mail: kodama@bme.tohoku.ac.jp \\ ${ }^{* * *}$ Faculty of Education, Hokkaido University of Education, Japan,

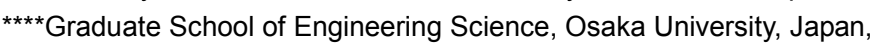

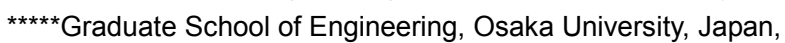 \\ ${ }^{* * * \star * *}$ Graduate School of Engineering, Hokkaido University, Japan
}

\begin{abstract}
Molecular delivery using ultrasound (US) and nano/microbubbles (NBs), i.e., sonoporation, has applications in gene therapy and anticancer drug delivery. When NBs are destructed by ultrasound, the surrounding cells are exposed to mechanical impulsive forces generated by collapse of either the NBs or the cavitation bubbles created by the collapse of NBs. In the present study, experimental, theoretical and numerical analyses were performed to investigate cavitation bubbles mediated molecular delivery during sonoporation. Experimental observation using lipid NBs indicated that increasing US pressure increased uptake of fluorescent molecules, calcein (molecular weight: 622), into 293T human, and decreased survival fraction. Confocal microscopy revealed that calcein molecules were uniformly distributed throughout the some treated cells. Next, the cavitation bubble behavior was analyzed theoretically based on a spherical gas bubble dynamics. The impulse of the shock wave (i.e., the pressure integrated over time) generated by the collapse of a cavitation bubble was a dominant factor for exogenous molecules to enter into the cell membrane rather than bubble expansion. Molecular dynamics simulation revealed that the number of exogenous molecules delivered into the cell membrane increased with increasing the shock wave impulse. We concluded that the impulse of the shock wave generated by cavitation bubbles was one of important parameters for causing exogenous molecular uptake into living cells during sonoporation.
\end{abstract}

Key words: Nanoparticles, Membrane Permeabilization, DDS, Fluorescence

\section{Introduction}

$\mathrm{Nano} /$ microbubbles (NBs) are encapsulated gas bubbles with a radius between $50 \mathrm{~nm}$ and $5 \mu \mathrm{m}$. These bubbles oscillate nonlinearly in an ultrasound (US) field and emit acoustic signals with harmonic and subharmonic components, on the basis of which their acoustic scattering and vasculature signatures are distinguished ${ }^{(1)}$. The shell membrane of NBs is composed of albumin, lipid, or polymer. The gas inside the bubble is either air or perfluorocarbons. Large molecules such as $\mathrm{C}_{3} \mathrm{~F}_{8}$ prolong enhancement time because of decreased diffusion ${ }^{(2)(3)}$. Ligands that are able to bind disease-related markers can be incorporated on the surface of the bubbles; thus, the bubbles can have an active function to move toward the target sites ${ }^{(4)}$. Several engineered bubbles that are aimed at targeting inflammation, angiogenesis, early tumor formation, and thrombi have been reported in the 
literature ${ }^{(5)}$.

NBs have been used to modulate targeted molecular mediators. Conceptually, NBs are mixed with exogenous molecules such as therapeutic genes or anticancer drugs and injected either locally or systemically. Targeted gene transfer is then achieved by destructing NBs located in a selective defined area ${ }^{(6)(7)(8)}$. The mechanical index $(M I)$ for destructing NBs is reported to be $0.1-0.5^{(9)(10)(11)(12)}$, where $M I$ is defined as the peak negative pressure divided by the square root of the US frequency. The efficiency of molecular delivery depends on the US parameters (exposure time, intensity, pulse length, and duty cycle of US) ${ }^{(13)(14)}$, the membrane components of the NBs ${ }^{(15)(16)}$, and the cell-to-NB ratio ${ }^{(17)(18)}$.

Collapse of NBs generates second products such as many tiny bubbles as well as debris that have gas attached or included as modeled by Harvey et al. ${ }^{(19)}$. Since NBs and the second products behave as cavitation nuclei ${ }^{(20)(21)(22)}$, cavitation bubbles generate from them in a field of US. Broadband noise measurements detected the generation of cavitation bubbles in the presence of US with varying pressures from 0.5 to $2.0 \mathrm{MPa}^{(23)}$.

NBs and cavitation bubbles generate mechanical forces such as bubble expansion, microstreaming, liquid jet impact, and shock waves. These forces interact with the surrounding cells, resulting in transient membrane permeability, followed by the entry of exogenous molecules ${ }^{(24)(25)(26)}$.

The pressure profile of a shock wave indicates its energy content, and shock-wave propagation in tissue is associated with cellular displacement, leading to the development of cell deformation. The efficiency of molecular delivery depends on the molecular size of exogenous molecules ${ }^{(27)(28)}$, and the application time of shock waves ${ }^{(28)(29)}$. Kodama et al. ${ }^{(30)}$ reported that the impulse of the shock wave (i.e., the pressure integrated over time) was a dominant factor for membrane permeability. Molecular dynamics (MD) simulation has shown that shock wave impulse induces water molecule penetration ${ }^{(31)(32)}$ and formation of transient water pores in a lipid bilayer ${ }^{(33)}$.

In the present study, experimental, theoretical and numerical analyses were performed to investigate cavitation bubbles mediated molecular delivery during sonoporation. The impulse of the shock wave generated by cavitation bubbles was shown as one of important parameters for causing exogenous molecular uptake into living cells in sonoporation.

\section{Materials \& Methods}

\subsection{Experiment}

Ultrasound

US was generated at $1.0 \mathrm{MHz}$ by using a 12 -mm-diameter submersible piezoceramic transducer (Fuji Ceramics Co., Tokyo, Japan) in a test chamber (300 $\times 450 \times 300 \mathrm{~mm}, \mathrm{~L} \times \mathrm{W} \times \mathrm{H}$ ) filled with tap water. A $1.0-\mathrm{MHz}$ sine wave (sinusoid) was generated using a multifunction synthesizer (WF1946A; NF Co., Yokohama, Japan) and amplified with a high-speed bipolar amplifier (HSA4101; NF Co.). The pressure values were measured using a PVDF needle hydrophone (PVDF-Z44-1000; Specialty Engineering Associates, Soquel, CA, USA) at a standoff distance of $1 \mathrm{~mm}$ from the transducer surface. The signals from the hydrophone were amplified and recorded into a digital oscilloscope $(500 \mathrm{MHz}, 1$ M $\Omega$ (16 pF), Wave Surfer 454; LeCroy Co., Chestnut, NY, USA). The peak positive acoustic pressures were $0.2,0.3$, and $0.5 \mathrm{MPa}$, and each pressure corresponded to the calculated acoustic intensity of $1.3,3.0$, and $8.3 \mathrm{~W} / \mathrm{cm}^{2}$, respectively. The intensity was defined as the average rate of energy flow through a unit area normal to the direction of propagation. $M I$ was $0.2,0.3$, and 0.5 , respectively. The values of the peak positive pressures were the same as those of the peak negative pressures. The duty ratio was $50 \%$; number of pulses, 2000 ; 
pulse repletion frequency (PRF), $250 \mathrm{~Hz}$; and exposure time, $10 \mathrm{~s}$.

Nano/microbubbles

Lipid NBs were created in an aqueous dispersion of $2 \mathrm{mg} / \mathrm{mL}$ 1,2-distearoyl-sn-glycero-3-phosphocholine (Avanti Polar Lipids, Alabaster, AL, USA) and $1 \mathrm{mg} / \mathrm{mL}$ polyethylene glycol 40 stearate (Sigma-Aldrich Co., St. Louis, MO, USA) by using a 20-kHz sonicator (Vibra Cell ${ }^{\mathrm{TM}}$; Sonics \& Materials, Inc., Danbury, CT, USA) in the presence of $\mathrm{C}_{3} \mathrm{~F}_{8}$ gas. The presence of lipid molecules in the lipid NB surface was confirmed by staining with $3 \mu \mathrm{M}$ FM1-43 (excitation: $553 \mathrm{~nm}$, emission: $570 \mathrm{~nm}$; Molecular Probe Inc., Eugene, OR, USA) and observation under an inverted microscope (IX81; Olympus Co., Tokyo, Japan). The mean concentration was $3.4 \times 10^{8}$ bubbles $/ \mathrm{mL}$. The peak diameters expressed in terms of size distribution and zeta potential were $1272 \pm$ $163 \mathrm{~nm}(n=7$, mean \pm S.D.) and $-4.11 \pm 0.74 \mathrm{mV}(n=4$, mean \pm S.D.), respectively (Fig. 1). Both values were measured by using a laser diffraction particle size analyzer (particle range of $0.6 \mathrm{~nm}-7 \mu \mathrm{m}$, ELSZ-2; Otsuka Electronics Co. Ltd, Osaka, Japan) in phosphate-buffered saline without $\mathrm{Mg}^{2+}$ and $\mathrm{Ca}^{2+}$ (PBS, $\mathrm{pH} 7.2$ at room temperature, Sigma-Aldrich) ${ }^{(34)}$.

\section{Cell culture}

In vitro studies were performed in accordance with the ethical guidelines of Tohoku University. Human embryonic kidney (293T) cells were obtained from Prof. M. Ono (Tohoku University, Japan) and were cultured in Dulbecco's modified Eagle's medium (DMEM) containing $10 \%$ fetal bovine serum and $1 \%$ penicillin-streptomycin in $250 \mathrm{~mL}$ culture flasks in a cell culture incubator (SCI-325D; Astec Co., Fukuoka, Japan) at $37^{\circ} \mathrm{C}$ under an atmosphere of $5 \% \mathrm{CO}_{2}$ in air. Prior to the US-mediated delivery experiments, the total cell counts and viability were determined using a hemocytometer with the trypan blue dye exclusion method ${ }^{(35)}$. Only cells that were in the exponential growth phase and had a viability of $\geq 99 \%$ were used.

\section{Fluorophores}

Calcein (622 Da) (excitation: $496 \mathrm{~nm}$, emission: $514 \mathrm{~nm}$; Sigma-Aldrich Co.) was used for the evaluation of the uptake of molecules by the cells in the presence of US and NBs. Calcein was dissolved in PBS and used at a concentration of $200 \mu \mathrm{M}$ in a complete medium. Stokes radius for calcein was estimated to be $0.68 \mathrm{~nm}^{(30)}$.

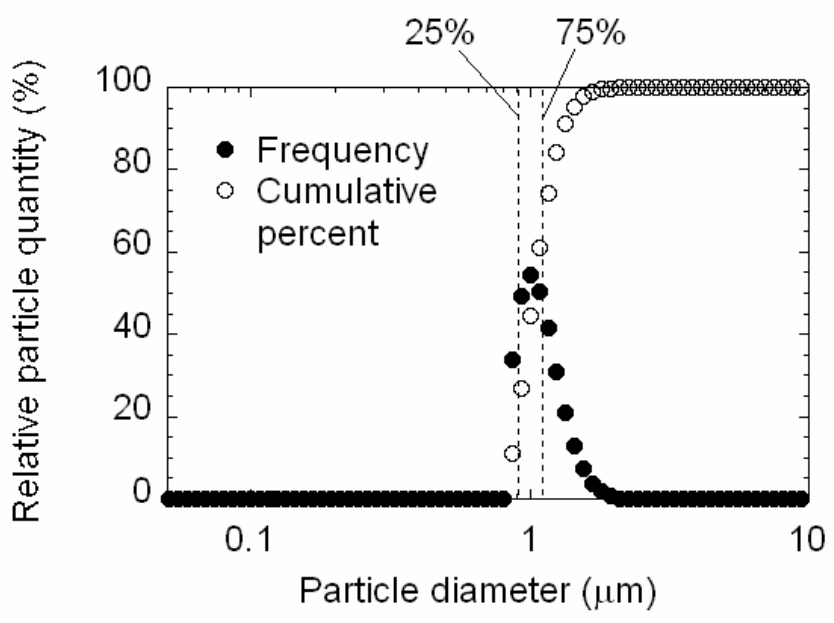

Fig. 1 Size distribution of lipid NBs. The mean peak of size distribution was $1.27 \pm 0.431 \mu \mathrm{m}(n=7$, mean \pm S. D.). $\bigcirc$ : cumulative percent,

: frequency, $n$ : number of samples. 


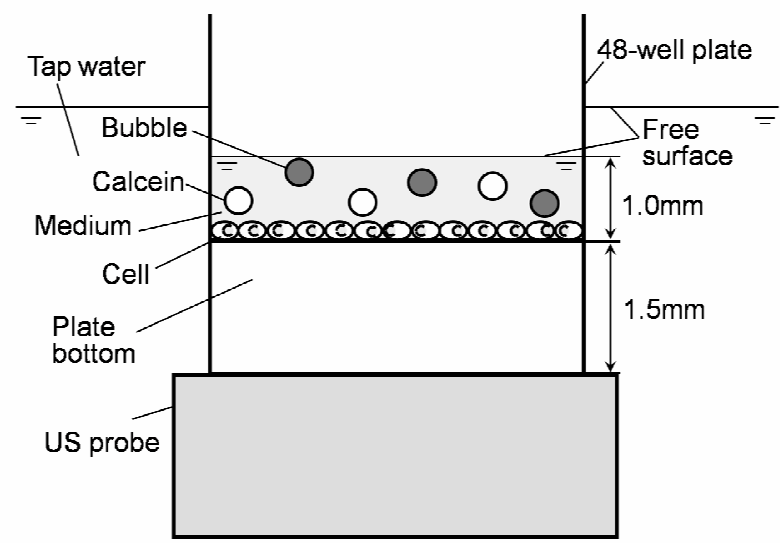

Fig. 2 Experimental setup. The 48-well culture plates were positioned just above the 1-MHz US probe in a test chamber $(300 \times 450 \times 300 \mathrm{~mm}, \mathrm{~L} \times \mathrm{W} \times \mathrm{H})$ filled with water. The basal plate thickness of the 48 -well culture plate was $1.5 \mathrm{~mm}$. By assuming the speed of sound in water to be $1500 \mathrm{~m} / \mathrm{s}$, we calculated the wavelength as $1.5 \mathrm{~mm}$. The height of the medium containing calcein and lipid NBs in the plate was $1 \mathrm{~mm}$. Complexed superimposed ultrasonic waves were generated in the medium. Atomized particles were generated with increasing US intensity, indicating the generation of cavitation bubbles.

\section{Ultrasound exposure}

Cells were seeded onto 48 -well plates alternately at $5 \times 10^{4}$ cells/well in a complete medium and incubated at $37^{\circ} \mathrm{C}$ in a $5 \% \mathrm{CO}_{2}$ incubator. After a $24-\mathrm{h}$ attachment period, the seeded cells were washed with PBS, and the medium was replaced with a fresh medium $(110 \mu \mathrm{L})$ containing calcein $(200 \mu \mathrm{M})$ with and without NBs $(10 \% \mathrm{v} / \mathrm{v})$. The height of the medium containing calcein and lipid-micelle bubbles in the plate was $1 \mathrm{~mm}$. The maximum geometrical characteristic radius $r_{0}$ occupied uniformly by a single NB (eqn (A2)) for a lipid bubble was calculated to be $19 \mu \mathrm{m}$. The US transducer was placed in the test chamber filled with tap water, and the plates were positioned just above the US probe (Fig. 2). The basal plate thickness of the 48 -well culture plate was $1.5 \mathrm{~mm}$. When the speed of sound in water was assumed to be $1500 \mathrm{~m} / \mathrm{s}$, the wavelength was calculated to be $1.5 \mathrm{~mm}$. Superimposed ultrasonic waves were generated in the media, and atomized particles were generated with increasing US intensity. The generation of particles indicated the generation of cavitation bubbles, as mentioned in the Results and Discussion section. The cells were exposed to US with a pressure of $0.2,0.3$, and $0.5 \mathrm{MPa}$ at a duty ratio of $50 \%$ for $10 \mathrm{~s}$. The number of cycles in the pulse was 2000. Twenty-four wells of the 48-well plate were used for each condition. Since the cells were seeded onto alternate wells, the neighboring wells were not exposed to US at the same time. Cell viability was determined using the MTT (3-[4,5-dimethylthiazol-2-yl]-2,5-diphenyltetrazolium bromide) assay as described previously ${ }^{(36)}$, and the cell survival fractions were expressed relative to cells not treated with NBs and US (control).

\section{Fluorescence measurement}

After the US exposure, supernatants from 6 wells of the 48-well plate were collected in a $15-\mathrm{mL}$ centrifuge tube. The cells in the wells were washed with $500 \mu \mathrm{L}$ PBS, and the PBS containing the cells was transferred to the abovementioned centrifuge tubes. All the cells were trypsinized $(100 \mu \mathrm{L} /$ well $)$ and transferred to each centrifuge tube. The cells were washed with PBS $(14 \mathrm{~mL}, 3 \times)$ to remove the excess extracellular fluorophore by centrifugation $(5 \mathrm{~min}$ at $350 \times \mathrm{g}$ ). Then, $1 \mathrm{~mL}$ PBS was added to the centrifuge tubes, and the cells were transferred to an eppendorf tube and centrifuged at $8000 \times g$ for $5 \mathrm{~min}$. After the supernatant was discarded, the pellet was lyzed with $200 \mu \mathrm{L}$ of reporter lysis buffer 
(Promega, Madison, WI, USA) and subsequently frozen at $-80^{\circ} \mathrm{C}$ for $15 \mathrm{~min}$. The cells were defrosted on ice. Each lysate was centrifuged at $12000 \times g$ for 2 min to pellet cell debris. Twenty microliters of the lysate was analyzed for the uptake of fluorescent molecules by using an Mx3000P ${ }^{\mathrm{TM}}$ (Stratagene, La Jolla, CA, USA). The fluorescence was excited using a quartz tungsten halogen lamp (350-750 nm), and the emission was collected through a band-pass filter of $492-516 \mathrm{~nm}$. The fluorescence data was analyzed with MxPro $^{\mathrm{TM}}$ QPCR Software (Stratagene). The total protein content in an aliquot of each sample supernatant was calculated with albumin standard curves (BCA protein assay kit; Pierce, Rockford, IL, USA). Two additional standard curves were utilized; one for the cell number and its total protein content, and the other for fluorescence concentration and its fluorescence intensity. Duplicates of the samples and standards were used for the experiment, and the absorption of the protein was measured at $562 \mathrm{~nm}$ by using a plate reader (Sunrise; Tecan Austria GmbH, Salzburg, Austria) with the data analysis software LS-PLATEmanager RD (Windows) 2001 (Sunrise). The number of equivalent fluorescent molecules per cell was determined from the calibration curves.

\section{Confocal fluorescence microscopy}

$293 \mathrm{~T}$ cells $\left(5 \times 10^{4}\right.$ cells/well $)$ were seeded in a complete medium in alternate 48 wells to prevent the exposure of neighboring cells to US. The medium was replaced on the next day with a fresh medium $(110 \mu \mathrm{L})$ containing calcein $(200 \mu \mathrm{M})$ with and without NBs $(10 \% \mathrm{v} / \mathrm{v})$. After an US exposure of $10 \mathrm{~s}$, the plates were incubated for $24 \mathrm{~h}$. The cells were then washed 3 times with PBS and trypsinized. Finally, the cell pellet was resuspended in $60 \mu \mathrm{L}$ of $0.7 \mu \mathrm{g} / \mathrm{mL}$ propidium iodide (PI, excitation: $535 \mathrm{~nm}$, emission: $617 \mathrm{~nm}$, Molecular Probes) and incubated at room temperature for 10-15 min. Confocal fluorescence microscopy was performed using a confocal microscope (FV1000, Olympus). A 60× oil-immersion objective lens with a numerical aperture of 1.25 was used. Calcein and PI fluorescence was excited with a 488-nm line of an argon laser. The laser excitation beam was directed to the specimen through a 488-nm dichroic beam splitter. Emitted fluorescence was collected through a 510- to 550-nm band-pass emission filter for the green channel and a 580-nm long-pass filter for the red channel. Computer-generated images of $1-\mu \mathrm{m}$ optical sections were obtained at the approximate geometric center of the cell as determined by repeated optical sectioning.

\section{Statistical analysis}

All the measurement values are expressed as either mean \pm S.D. (standard deviation) or mean \pm S.E.M. (standard error of means). Statistical analysis for the calcein uptake was performed by Kruskal-Wallis test. When the Kruskal-Wallis test was significant, the differences between each group were estimated using the Scheff's $F$ test as a post-hoc procedure. The differences were considered to be significant at $P<0.01$. For the survival fraction, Bartlett test was performed followed by one-way analysis of variance (ANOVA). When the one-way ANOVA was significant, the differences between each group were estimated using the Tukey-Kramer test as a post-hoc procedure. The differences were considered to be significant at $P<0.05$ or $P<0.01$.

\subsection{Theory}

Exposure of cells to US in the presence of NBs generates atomized particles on the surface, indicating the generation of cavitation bubbles ${ }^{(26)}$. In the medium, NBs and cavitation bubbles interact with each other, and the resulting complex physical forces such as shear stress, liquid jet impact, and shock waves may affect the cell membrane ${ }^{(23)(26)(37)(38)(39)}$. A further investigation of individual physical parameters would lead us into that specialized area of non-spherical bubble dynamics near cell surfaces and 
mechanisms of impulsive pressures, and such a digression may shift the focus of our argument. Therefore, we will concentrate on the behavior of a spherical cavitation bubble in an US field and the resulting shock wave phenomenon against the cell.

Cavitation bubble behavior depends on the properties of the surrounding boundaries ${ }^{(40)(41)}$. The critical relative distance affecting the cavitation bubble behavior is defined as $L / R_{\max }$, where $L$ is the distance between the boundary and the bubble inception position and $R_{\max }$ is the maximum bubble expansion radius. When NBs are distributed uniformly in the medium and each NB produces a single cavitation bubble at $L / R_{\max }>4{ }^{(42)}$, the bubble motion can be analyzed spherically.

\section{Bubble dynamics}

We assume that the US contrast agents are destroyed by US and the internal gas $\mathrm{C}_{3} \mathrm{~F}_{8}$ is trapped in the crevices of the debris of the destroyed agents; additionally, the cavitation bubbles are assumed to be generated from the gas trapped in the crevices. Furthermore, we consider the gas as cavitation nuclei, as modeled by Harvey et al. ${ }^{(19)}$. We assume that spherical cavitation bubbles with a nanometer diameter size can be analyzed as a continuum. The motion of a free spherical cavitation bubble is given by the Keller-Miksis equation ${ }^{(43)}$ as shown below. This equation is corrected to the first order of the Mach number of the bubble wall motion. The error term is the order $\left(\dot{R} / C_{L}\right)^{2}{ }^{(44)}$.

$$
\ddot{R}\left(1-\frac{1}{C_{L}} \dot{R}\right)+\frac{3}{2} \dot{R}^{2}\left(1-\frac{1}{3 C_{L}} \dot{R}\right)=\left(1+\frac{\dot{R}}{C_{L}}\right) \frac{1}{\rho_{L}}\left[P_{r=R}(t)-P_{C}\left(t+\frac{R}{C_{L}}\right)-P_{0}\right]+\frac{R}{\rho_{L} C_{L}} \frac{d P_{r=R}(t)}{d t}
$$

the pressure $P_{r=R}$ at the bubble surface is given by the equation:

$$
P_{r=R}(t)=\left(P_{0}+\frac{2 \sigma_{L}}{R_{0}}\right)\left(\frac{R_{0}}{R}\right)^{3 r}-\frac{2 \sigma_{L}}{R}-\frac{4 \mu_{L}}{R} \dot{R}
$$

the oscillation pressure $P_{\mathrm{C}}$ is given by the equation:

$$
P_{C}(t)=\left|P_{A}\right| \sin \omega t
$$

where $R$ is the radius of the bubble; $C_{\mathrm{L}}$, sound velocity in liquid $(1497 \mathrm{~m} / \mathrm{s}) ; \rho_{\mathrm{L}}$, density of water $\left(997 \mathrm{~kg} / \mathrm{m}^{3}\right) ; P_{0}$, atmospheric pressure $(101.3 \mathrm{kPa}) ; R_{0}$, the initial bubble radius; $\gamma$, adiabatic exponent of a gas $(1.07){ }^{(45)} ; \mu_{\mathrm{L}}$, the liquid shear viscosity $(0.89 \mathrm{mPa} \cdot \mathrm{s}) ; \sigma_{\mathrm{L}}$, surface tension of water $(72 \mathrm{mN} / \mathrm{m}) ; P_{A}$, peak positive pressure measured in the experiments; and $\omega$, the circular frequency. Thermal diffusion at the bubble wall and noncondensable gas were ignored.

When a cavitation bubble collapses, a spherical shock wave is emitted as the bubble rebounds. The shock wave interacts with the surrounding cells, resulting in cell membrane damage. The distance of the shock wave propagation from the center of the bubble $r_{\mathrm{C}}$ required to damage to the cell membrane is given by the equation ${ }^{(46)}$ :

$$
r_{C} \approx \frac{P_{\max } R_{\min }}{\varepsilon_{C} \rho_{L} C_{L}^{2}}
$$

where $P_{\max }$ is the maximum pressure when the bubble reaches the minimum radius $R_{\min }$ and $\varepsilon_{\mathrm{C}}$ is the static critical strain required to damage the membrane. $\varepsilon_{\mathrm{C}}$ is estimated to be $0.02-0.03$ for the red blood cell membrane ${ }^{(47)}$. 
The shock wave pressure $P \mathrm{~s}$, defined as the peak value of the shock wave pressure, decreases as approximately $1 / r_{S}$ while it propagates outward ${ }^{(48)}$; thus, $P \mathrm{~s}$ is given by the equation:

$$
P_{S}=\frac{P_{\max } R_{\min }}{r_{S}}
$$

where $r_{S}$ is the radial distance of the shock front from the origin.

The acoustic energy $E$ s of a spherical shock wave is given by the equation ${ }^{(49)}$ :

$$
E_{S}=\frac{4 \pi r_{S}^{2}}{\rho_{L} C_{L}} \int_{0}^{t^{\prime}} P(t)^{2} d t
$$

By assuming that the shock wave has wave characteristics that vary in the form of exponential decay with respect to time, we defined the pressure profile $P(\mathrm{t})$ as follows:

$$
P(t) \cong P_{s} \exp \left[\frac{-t \cdot \ln 2}{t_{s}}\right]
$$

where $P \mathrm{~S}$ is the peak pressure and $t_{\mathrm{S}}$ is the full width at half-maximum (FWHM) pulse duration of a shock wave (i.e., the time satisfying the condition $\left.\left(P_{\mathrm{S}}+P_{0}\right) / 2\right)$, which is numerically obtained. From eqn (6), Es is given as follows ${ }^{(50)}$ :

$$
E_{S}=\frac{2 \pi R_{\min }^{2} P_{\max }^{2} t_{s}}{\rho_{C} C_{L} \ln 2}
$$

On the assumption that $E \mathrm{~s}$ is conserved during the shock wave propagation, $t_{\mathrm{rC}}$ at $r=r_{\mathrm{C}}$ is described as follows:

$$
t_{r_{c}}=\frac{\rho_{L} C_{L} \ln 2 E_{S}}{2 \pi r_{C}^{2} P_{\max }^{2}}
$$

The shock wave impulse $I$ at $r=r_{\mathrm{C}}$ is given as follows:

$$
I_{r_{c}}=\frac{\rho_{L} C_{L} E_{S}}{2 \pi r_{C} R_{\text {min }} P_{\text {max }}}
$$

The equ (1) was calculated up to 50 periodic times using a fourth-order Runge-Kutta method to determine the maximum pressure $P_{\max }$, maximum bubble radius $R_{\max }$, and minimum bubble radius $R_{\min }$, respectively. The chaotic behavior of the bubble motion due to the nonlinear oscillation was not considered.

\subsection{Molecular dynamics simulation}

\section{Modeling of lipid bilayer}

The cell membrane is a thin film (approximately $5 \mathrm{~nm}$ ) composed of lipids and proteins. The lipid bilayer forms the basic structure of the membrane, while the protein molecules exist as dissolved entities in this layer. Four major phospholipids predominate in the plasma membrane of many mammalian cells: phosphatidylcholine (DPPC), sphingomyelin, phosphatidylserine, and phosphatidylethanolamine ${ }^{(51)}$. Following the study of Koshiyama 
et al. ${ }^{(31)}$, we calculated the interaction of a single lipid bilayer with a shock wave. The lipid bilayer was designed as a 32 DPPC lipid bilayer sandwiched between 2 layers of 2400 water molecules in a rectangular calculation box. The long axis ( $z$ axis) of the rectangular box was perpendicular to the bilayer plane ( $x y$ plane). The water molecules were calculated using a simple point charge (SPC) model, and all the bond interactions between the atoms in the DPPC molecules were calculated. The stable liquid-crystal phase bilayer was calculated for several tens of nanoseconds under a constant temperature of $50^{\circ} \mathrm{C}$ and a pressure of $101.3 \mathrm{kPa}$ with periodic boundary conditions.

\section{Shock wave impulse}

The shock wave impulse per unit area $I$ is defined as follows ${ }^{(30)}$ :

$$
I=\int_{0}^{t_{+}} p(t) d t
$$

where $t$ is the time; $p(t)$, the pressure near the cells in water; and $t_{+}$, the positive phase duration of a half cycle of the shock wave ${ }^{(30)}$. On the basis of the definition of impulse, the shock impulse $I$ can be regarded as the increment in the momentum of water divided by an area $A$ (the cross-sectional area normal to the $z$ direction of the simulation box) on which the pressure $p(t)$ is exerted. The shock wave impulse is given as follows:

$$
I=\frac{M\left(t_{+}\right)-M(0)}{A}
$$

where $M(t)$ is the momentum of water at time $t$. At time $t=0$, the shock wave did not reach the cells, and the water molecules in the front of the shock wave were at rest; therefore, $M(0)=0$. When $t=t_{+}$, the shock wave passed over a small volume of water near the cells, and the momentum $M\left(t_{+}\right)=I \times A$ was transferred to the small volume of water. At the beginning of shock wave simulation, the momentum $M\left(t_{+}\right)$was applied to water molecules adjacent to the bilayer in a volume $A \times L_{\mathrm{Z}}$, where $A=3.77 \mathrm{~nm} \times 2.72 \mathrm{~nm}=10.25 \mathrm{~nm}^{2}$ and $L_{Z}$ is the length of the volume of water in the $z$ direction ${ }^{(31)}$. The choice of $L_{Z}$ is arbitrary, and we set $L_{Z}=4 \mathrm{~nm}$, which is almost equal to the initial thickness of the bilayer. This is because the present simulation was focused on the behavior of the bilayer with the excess momentum $M\left(t_{+}\right)$added by the shock wave. The change in the momentum of the water molecules at the beginning of the shock simulation was numerically implemented by the addition of an average velocity $V$ to the thermal velocity of the water molecules in the equilibrated bilayer/water system.

The average velocity $V$ is given by

$$
V=\frac{M\left(t_{+}\right)}{m N}=\frac{I \times A}{m N}
$$

where $m$ is the weight of a water molecule and $N(\sim 1000)$ is the number of water molecules in the volume $A \times L_{Z}$. The impulse $I$ is increased from 0 to $100 \mathrm{mPa} \cdot \mathrm{s}$ at an interval of 2.5 $\mathrm{mPa} \cdot \mathrm{s}$, and $V$ is then changed from 0 to $25600 \mathrm{~m} / \mathrm{s}$. The average velocity $V$ corresponds neither to the speed of sound in water nor to the propagation speed of the shock wave. It represents only the increase in the momentum of water molecules due to the shock wave. The modeling of the shock wave by the impulse enables qualitative comparison between the present numerical results and the previous experimental ones. 
(a)

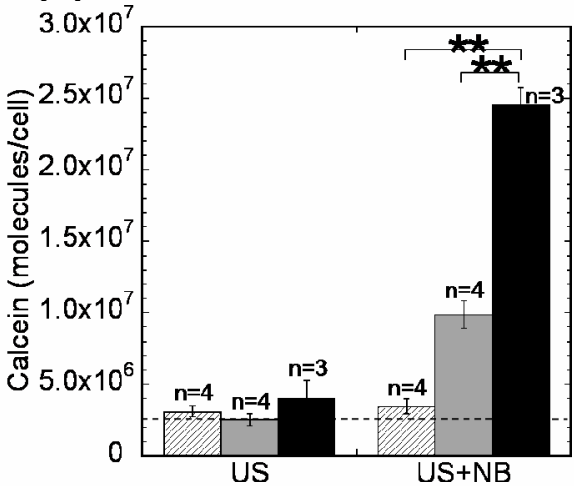

(b)

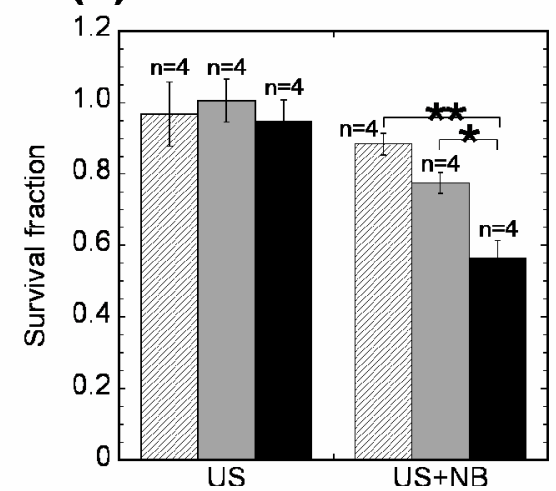

(c)
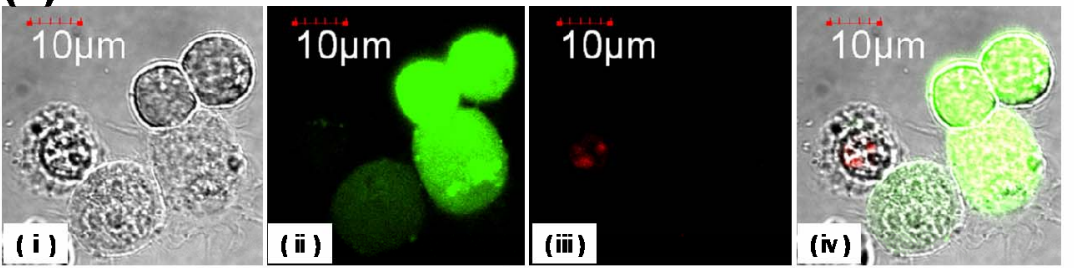

Fig. 3 Effects of NBs on the uptake of calcein (molecular weight: 622). 293T cells with and without NBs were exposed to US with varying US pressure $P_{\mathrm{A}}(0.2,0.3$, and $0.5 \mathrm{MPa})$. Duty ratio, $50 \%$; number of pulses, 2000; pulse repletion frequency (PRF), $250 \mathrm{~Hz}$; and exposure time, $10 \mathrm{~s}$. (a) The number of intracellular molecules per cell. The calcein uptake of the control samples that are without US $+\mathrm{NB}$ is indicated as the broken, which is similar to that of US alone. Values are expressed as means \pm S.E.M. $(n=$ $3-4)$, where $n$ is the number of samples. Statistical analysis for the calcein uptake was performed by Kruskal-Wallis test. When the Kruskal-Wallis test was significant, the differences between each group were estimated using the Scheff's $F$ test as a post-hoc procedure. The differences were considered to be significant at $P<0.01(* *)$. (b) Survival fractions determined by the MTT assay. Values are expressed as means \pm S.E.M. $(n=4)$. Statistical analysis was performed by Bartlett test followed by one-way analysis of variance (ANOVA). When the one-way ANOVA was significant, the differences between each group were estimated using the Tukey-Kramer test as a post-hoc procedure. The differences were considered to be significant at $P<0.05\left(^{*}\right)$ or $P<0.01(* *)$. Wh $: 0.2 \mathrm{MPa} \square: 0.3 \mathrm{MPa}, \mathbf{\square}: 0.5 \mathrm{MPa}$. (c) Confocal fluorescence microscopy showing (i) differential interference contrast, (ii) fluorescence images, (iii) representative viable $293 \mathrm{~T}$ cells exposed to US in the presence of NBs and (iv) color-merged image. In fluorescence staining, PI was used to confirm that the cells showing calcein uptake were viable. Scale bars = $10 \mu \mathrm{m}$. US pressure was $0.5 \mathrm{MPa}$; duty ratio, $50 \%$; number of pulses, 2000; pulse repletion frequency (PRF), $250 \mathrm{~Hz}$; and exposure time, $10 \mathrm{~s}$.

\section{Results and Discussion}

\section{Observation of cavitation bubbles}

First, we investigated the number of calcein molecules delivered into cells in the presence of NBs and US in the experimental system as seen in Fig. 2. The medium containing NBs was a white emulsified suspension. After exposure to US, the medium became transparent, and fragmented debris was found floating on the surface. Atomized particles were detected on the surface with increasing US pressure. In contrast, these particles were not observed with increasing US pressure in the absence of NBs. This phenomenon indicates that impurities in the medium enhance the generation of cavitation bubbles, resulting in the production of capillary waves and subsequent atomized particles ${ }^{(26)}$. The mean diameter $d$ of the atomized particles due to capillary waves generated by US on the free surface is determined by the liquid surface tension $\sigma_{\mathrm{L}}$, liquid density $\rho_{\mathrm{L}}$, and US frequency $f$, which is given by eqn (A1). Thus, the mean diameter of the atomized particles is calculated to be 7.9 $\mu \mathrm{m}$ for $1-\mathrm{MHz}$ US. The detailed mechanism of atomized particle generation has been reported by Yule and Al-Suleimani ${ }^{(52)}$. 

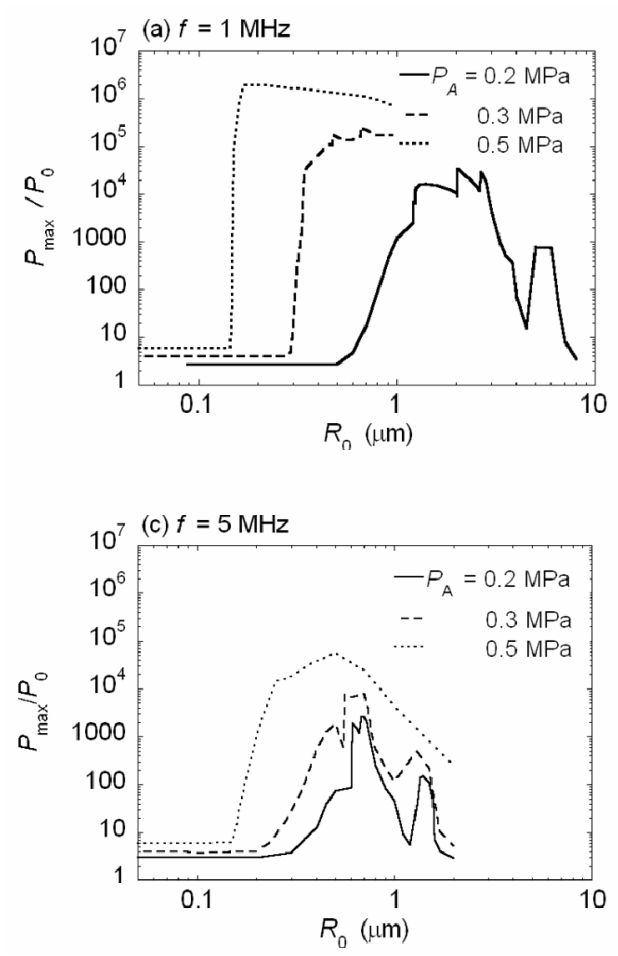

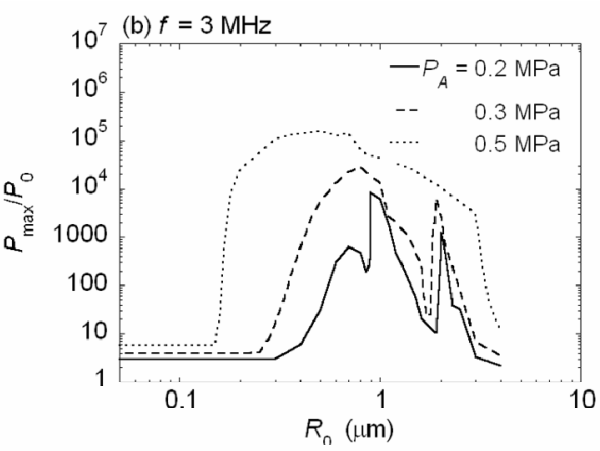

Fig. 4 The normalized maximum pressure $P_{\max } / P_{0}$ generated at the cavitation bubble wall at the minimum radius $R_{\min }$ as a function of $R_{0}$ for US pressure ( $P_{\mathrm{A}}=0.2,0.3$, and 0.5 $\mathrm{MPa})$ and US frequency $(f=1,3$, and 5 $\mathrm{MHz})$, where $P_{0}(=101.3 \mathrm{kPa})$ was the atmospheric pressure. Numerical data were obtained using a fourth-order Runge-Kutta method.

\section{Uptake of calcein into cells by sonoporation}

Figure 3 a shows the number of calcein molecules per cell with varying US pressure in the presence/absence of NBs. The duration of exposure to US was $10 \mathrm{~s}$. The calcein uptake of the control samples that are without US +NB is indicated as the broken, which is similar to that of US alone. The presence of the NBs caused a significant increase in the calcein uptake with increasing US pressure, resulting in the delivery of $2.5 \times 10^{7}$ calcein molecules per cell at $P_{\mathrm{A}}=0.5 \mathrm{MPa}(P<0.01)$. The increase in the uptake was associated with the increase in the generation of the atomized particles.

Figure $3 \mathrm{~b}$ shows the survival fraction of cells exposed to US with and without NBs measured by the MTT assay. US alone did not affect the survival fraction at these pressure values ${ }^{(26)}$; however, the survival fraction in the presence of NBs decreased with increasing pressure $(P<0.01)$. The uptake of exogenous molecules was inversely proportional to the survival fraction; this finding was in agreement with previous results ${ }^{(26)(53)}$. To confirm that the calcein molecules actually entered the cytoplasm, confocal fluorescence microscopy was performed. Figure 3c shows (i) the differential interference contrast, (ii) fluorescence images, and (iii) representative viable $293 \mathrm{~T}$ cells exposed to US $(0.5 \mathrm{MPa})$ in the presence of NBs and (iv) color-merged image. In some fluorescence staining, PI was used to confirm that the cells showing calcein uptake were viable and excluded PI (Fig. 3c(iii)). Some cells treated with US in the presence of NB showed intense fluorescence that was uniformly distributed throughout the entire cell.

\section{Motion of cavitation bubbles in the field of ultrasound}

From the experimental results, we assumed that cavitation bubbles were related to the transient membrane permeability and subsequent molecular uptake into cells. Next, we analyzed the behavior of a single spherical cavitation bubble in a wide range of parameters including the experimental conditions. Figure 4 shows the relationship between the normalized maximum pressure $P_{\max } / P_{0}$ generated on the surface of the cavitation bubble wall at the minimum radius $R_{\min }$, and the bubble initial radius $R_{0}$, where the atmospheric pressure $P_{0}$ was $101.3 \mathrm{kPa}$. $R_{0}$ was varied from 0.05 to $10 \mu \mathrm{m}$, US frequency $f$ was 1,3 , and $5 \mathrm{MHz}$, and the US pressure $P_{\mathrm{A}} 0.2,0.3$ and $0.5 \mathrm{MPa}$. Figure $4 \mathrm{a}$ is when $f=1 \mathrm{MHz}$. The 


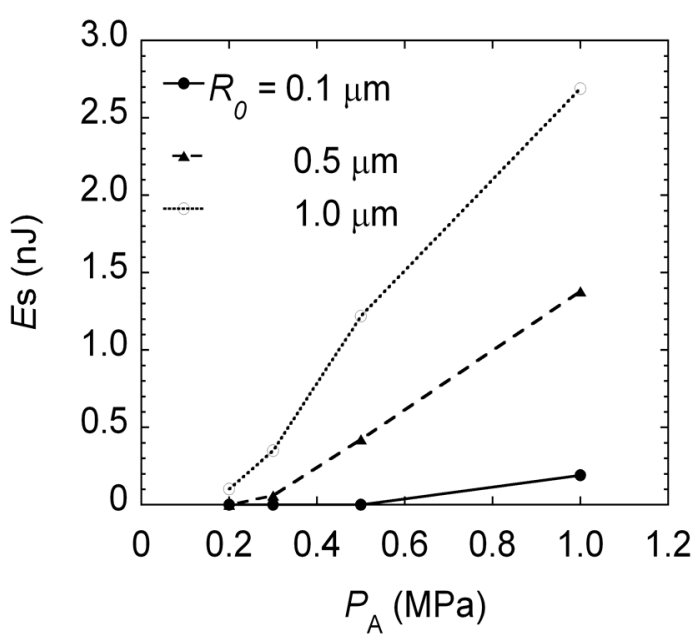

Fig. 5 Relationship between the shock wave energy Es given by eqn (8) and the US pressure $P_{A}$. The US frequency $f$ was $1 \mathrm{MHz}$. Numerical data were obtained using a fourth-order Runge-Kutta method.

$P_{\max } / P_{0}$ increased gradually with increasing $R_{0}$, and a peak was obtained at around $R_{0}=$ 1.2 3.0 $\mu \mathrm{m}$. After the first peak, $P_{\max } / P_{0}$ decreased rapidly, and the second peak was obtained at around $R_{0}=5 \sim 6 \mu \mathrm{m}$. These irregularities are due to the nonlinear cavitation bubble motion. The peak $P_{\max } / P_{0}$ increased with increasing $P_{\mathrm{A}}$, and its position shifted to lower values of $R_{0}$. When $R_{0}=0.635 \mu \mathrm{m}(=1.27 \div 2 \mu \mathrm{m})$ that is the initial radius of NBs used in the experiment, $P_{\max } / P_{0}=4.9 \rightarrow 1.3 \times 10^{6}$ when $P_{\mathrm{A}}=0.2 \rightarrow 0.5 \mathrm{MPa}$. In Fig. 3a, the uptake of calcein increases by a factor of 3.6 when $P_{\mathrm{A}}=0.2 \rightarrow 0.5 \mathrm{MPa}$. Thus, the increase in $P_{\max } / P_{0}$ related to the increase in the uptake, indicating that shock waves generated by cavitation bubbles were involved in the uptake of calcein.

Figures $4 \mathrm{~b}$ and $4 \mathrm{c}$ shows the profile of $P_{\max } / P_{0}$ when $f=3$, and $5 \mathrm{MHz}$, respectively. The $P_{\max } / P_{0}$ decreased when $f=1 \rightarrow 5 \mathrm{MHz}$, i.e. the generation of cavitation bubbles is suppressed with increasing US frequency. The fact that the threshold of generation of cavitation bubbles decreases with increasing frequency is in agreement with theoretical ${ }^{(54)}$ and recent experimental results obtained by broadband noise ${ }^{(23)}$. However, it should be noted that the pressure values were overestimated outside the range of values used for deriving equations.

Interaction of cell membrane with shock wave emitted from a cavitation bubble

When a cavitation bubble reaches its minimum radius $R_{\min }$, it expands and moves the surrounding liquid in the radial direction, thereby resulting in a pressure wave in the vicinity of the bubble wall. The wave propagates outward with a steep pressure front to become a shock wave.

Figure 5 shows the relationship between the shock wave energy $E$ s given by eqn (8) and the US pressure $P_{\mathrm{A}}$ at the US frequency $f$ of $1 \mathrm{MHz}$. The initial bubble radius $R_{0}$ varied from 0.1 to $1.0 \mu \mathrm{m}$. $E_{\mathrm{S}}$ increased with increasing $P_{\mathrm{A}}$ and $R_{0}$, where $E \mathrm{~s}$ was $2.7 \mathrm{~nJ}$ at $P_{\mathrm{A}}=1$ $\mathrm{MPa}$ and $R_{0}=1.0 \mu \mathrm{m}$.

The shock wave attenuates approximately proportional to $1 / r_{\mathrm{S}}\left(r_{\mathrm{S}}\right.$ : the radial distance of the shock front from the origin) ${ }^{(48)(55)(56)}$ and interacts with the surrounding cells, resulting in cell membrane damage. In addition, a rapid bubble radial expansion mechanically damages the surrounding cells. Figure 6 shows the shock wave propagation distances $\left(r_{\mathrm{C}}\right)$ from the point of its generation as a function of the initial bubble radius $R_{0}$ at the US frequency $f$ of 1 $\mathrm{MHz}$, where $R_{\max }$ is the calculated maximum bubble radius, and $\varepsilon_{\mathrm{C}}$ is necessary to disrupt the membrane. Evans et al. ${ }^{(47)}$ reported that $\varepsilon_{\mathrm{C}}$ was estimated to be $0.02-0.03$ for the red blood cell membrane. In Fig. 6, the shock wave can cause membrane damage at distances less than $r_{\mathrm{C}}$. The relationship of $r_{\mathrm{C}}>R_{\max }$ was satisfied for all values of $P_{\mathrm{A}}$ considered in the present study, i.e., the shock wave was regarded as the main factor of membrane damage rather than bubble expansion. From eqn (A2), the characteristic radius $r_{0}$ for a single lipid 

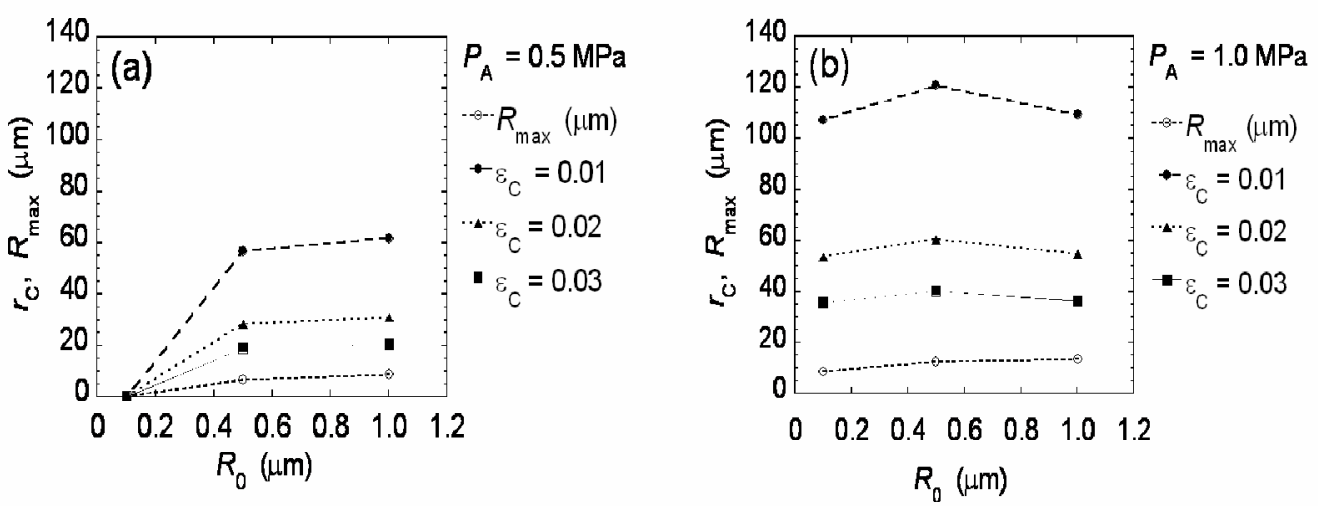

Fig. 6 Potential radius $r_{\mathrm{C}}$ of inducing cell damage by a shock wave from a cavitation bubble and the maximum bubble expansion radius $R_{\max }$, where $r_{\mathrm{C}}$ was given by eqn (4). The initial bubble radius $R_{0}$ was $0.1-1.0 \mu \mathrm{m}$. The critical strain $\varepsilon_{\mathrm{C}}$ was $0.01-0.03$. The US pressure $P_{\mathrm{A}}$ was $0.5-1.0 \mathrm{MPa}$. The US frequency $f$ was $1 \mathrm{MHz}$. Numerical data were obtained using a fourth-order Runge-Kutta method.

bubble was calculated to be $19 \mu \mathrm{m}$ prior to the US exposure. If a single NB produces multiple cavitation bubbles, the conditions $r_{\mathrm{C}}>r_{0}$ and $r_{\mathrm{C}}>R_{\max }$ are satisfied, i.e., the entire area of the medium can be regarded as the shock wave dominant area; therefore, all cells in the medium would be loaded by the shock waves created by the cavitation bubbles.

Figure 7 shows the relationship between $P_{\mathrm{A}}$ and the impulse $I r_{\mathrm{c}}$ at $r_{\mathrm{C}}$ with varying $\varepsilon_{\mathrm{C}}$, given by eqn (10) at the US frequency $f$ of $1 \mathrm{MHz}$. When $R_{0}=0.5 \mu \mathrm{m}$ (Fig. 7a), a peak of each characteristic line was obtained at $P_{\mathrm{A}}=0.3 \mathrm{MPa}$, and the value increased with increasing $\varepsilon_{\mathrm{C}}$. The peak was due to the bubble natural frequency. When $R_{0}=1.0 \mu \mathrm{m}$ (Fig. $7 b$ ), peaks were not observed in the figure; however, the lines decreased rapidly with increasing $P_{\mathrm{A}}$ to reach a constant value. This figure indicates that a spherical cavitation bubble with a radius of $0.5-1.0 \mu \mathrm{m}$ generates impulse values up to $100 \mathrm{mPa} \cdot \mathrm{s}$.

\section{MD simulation of water molecular delivery into the lipid bilayer with shock wave impulse}

It is desirable to calculate the number of calcein molecules delivered into cells with MD simulation for comparison with the experimental results. However, to our knowledge, there are no reliable calculation parameters such as force fields or partial charges to reproduce calcein thermodynamic properties and quantum factors. In the present study, we compared the number of water molecules delivered into the lipid bilayer calculated by MD simulation with that of calcein molecules obtained experimentally.

Figure 8 shows the relationship between the shock wave impulse and the penetration rate of water molecules into the bilayer obtained by MD simulation. The penetration ratio of water molecules into the lipid bilayer increased with increasing shock wave impulse. Water molecules were delivered into the cells at the rate of $0-0.3 \mathrm{molecules} /\left(\mathrm{ps} \cdot \mathrm{nm}^{2}\right)$ at $I=0-11$ $\mathrm{mPa} \cdot \mathrm{s}$ at $R_{0}=0.5 \mu \mathrm{m}$ and $\varepsilon_{\mathrm{C}}=0.03$ (Figs. 7a and 8). In contrast, at $R_{0}=1.0 \mu \mathrm{m}$ and $\varepsilon_{\mathrm{C}}=$ 0.03 (Figs. $7 \mathrm{~b}$ and 8 ), water molecules were delivered into the cells at a rate of $0.2-7.6$ molecules $/\left(\mathrm{ps} \cdot \mathrm{nm}^{2}\right)$ at $I=7.2-95 \mathrm{mPa} \cdot \mathrm{s}$.

Although it is reported that water molecules can penetrate into the cytoplasm across the membrane on macroscopic time scales ${ }^{(51)}$, the event rarely occurs on molecular time scales (below nanosecond time scales). However, in the present molecular simulation, water penetration was observed on picosecond time scales. Here, we assumed that at the cell radius $r$ of $5 \mu \mathrm{m}$, the shock wave impulse was loaded to a cell from above, and the duration of the impulse was $1 \mathrm{ps}$. The surface area of the cell hemisphere was given by $2 \pi r^{2}$, and the number of water molecules delivered into the lipid bilayer was calculated to be around $10^{7} \sim 10^{9}$. The radius of the water molecule was approximately $0.1 \mathrm{~nm}$, and the radius of calcein was estimated to be $0.68 \mathrm{~nm}^{(30)}$. Although the size of the calcein molecule was 

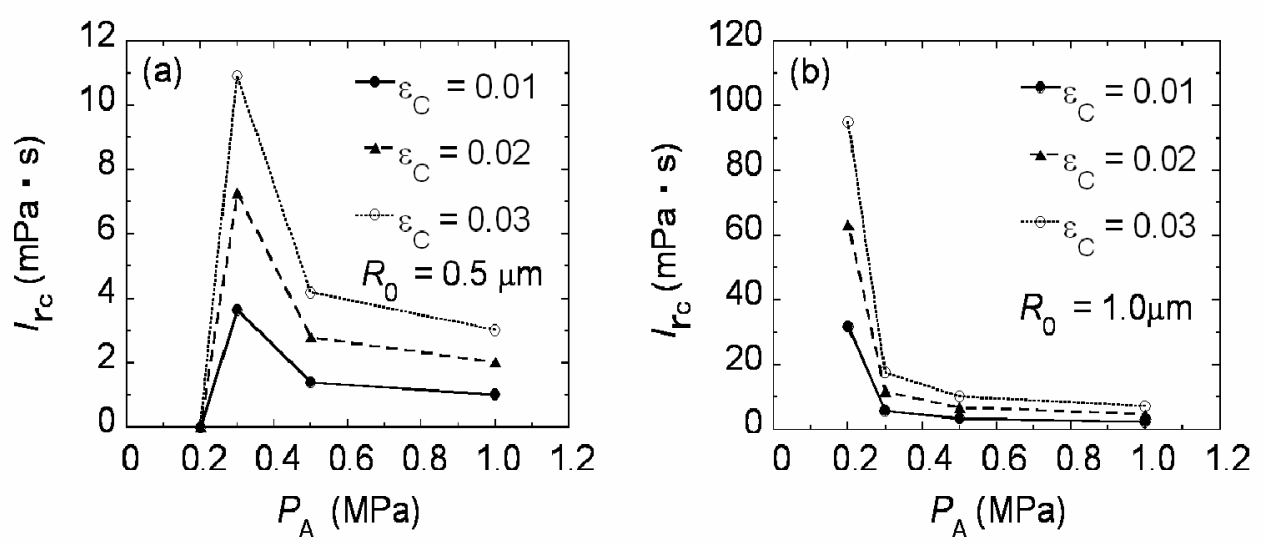

Fig. 7 Relationship between the shock wave impulse $\operatorname{Ir}_{\mathrm{C}}(\mathrm{mPa} \cdot \mathrm{s})$ and the US pressure $P_{\mathrm{A}}(0.2-1.0 \mathrm{MPa})$. The US frequency $f$ was $1 \mathrm{MHz}$. The critical strain $\varepsilon_{\mathrm{C}}$ was $0.01-0.03$. (a) $R_{0}=0.5 \mu \mathrm{m}$, (b) $R_{0}=1.0 \mu \mathrm{m}$. Numerical data were obtained using a fourth-order Runge-Kutta method.

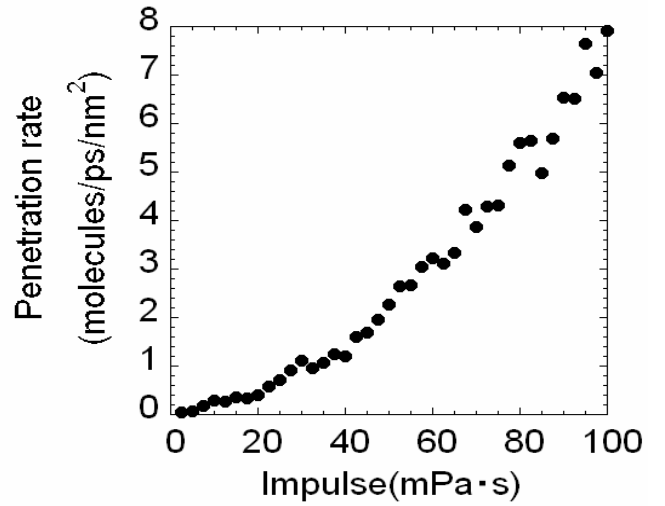

Fig. 8 Relationship between the shock wave impulse and the penetration ratio of water molecules calculated by MD simulation, in which the penetration ratio was calculated in the hydrophobic region at the instant of termination of simulation divided by the cross-sectional area $\left(A=10.25 \mathrm{~nm}^{2}\right)$ and the simulation time.

larger than that of the water molecule by a factor of 6.8 , the number of delivered calcein molecules in the order of $10^{7}$ per cell (Fig. 3) corresponded to the numerical values. We should note that the estimation of calcein delivery is on experimental time scales (several seconds have elapsed after exposure to ultrasound). On these time scales, the number of delivered water molecules might become considerably larger than that of calcein molecules because many shock waves are generated by pulsating bubbles' impact on membranes. At present, we have investigated water pore formation in lipid bilayers induced by the shock wave impulse ${ }^{(33)}$. This study suggests that more water penetration induces more larger structural changes in the lipid bilayer. Although it is not possible to estimate the number of water molecules that penetrate into the cells after induction by shock waves by using any other method, the results of the experimental estimation of calcein and numerical estimation of water molecules in the present study imply that the penetration of a large number of water molecules is required before the entry of large molecules. Because water penetration depends on the shock wave impulse generated in the conditions studied here, we believe that the impulse of the shock waves generated by the cavitation bubbles is one of the important parameters for permeabilization during sonoporation.

\section{Conclusion}

In the present study, experimental, theoretical and numerical analyses were performed to investigate cavitation bubbles mediated molecular delivery during sonoporation. The following conclusions were obtained.

1. Experimental observation using lipid nano/microbubbles indicated that increasing US 
pressure increased uptake of fluorescent molecules, calcein, into 293T human, and decreased survival fraction.

2. Theoretical analysis based on a spherical gas bubble dynamics indicated that the impulse of the shock wave (i.e., the pressure integrated over time) generated by the collapse of a cavitation bubble created by nano/microbubbles was one of dominant factors for exogenous molecules to enter into the cell membrane rather than bubble expansion.

3. Molecular dynamics simulation revealed that the number of exogenous molecules delivered into the cell membrane increased with increasing the shock wave impulse.

4. The impulse of the shock wave generated by cavitation bubbles was one of important parameters for causing exogenous molecular uptake into living cells during sonoporation.

\section{Acknowledgments}

TK acknowledges the Grant-in-Aid for Scientific Research (B) (20300173); the Grants-in-Aid for Scientific Research on Priority Area, MEXT (20015005); and the Grant for Research on Advanced Medical Technology, the Ministry of Health, Labour and Welfare of Japan (H19-nano-010). YT acknowledges the Grant-in-Aid for Scientific Research (C) (20560144), and KK acknowledges the Grant-in-Aid for Young Scientists (B) ( 207601140004).

\section{Appendix}

Atomized liquid particles generated with US

The mean diameter $d$ of the atomized particles due to capillary waves generated by US on the free surface is given by the equation ${ }^{(57)}$ :

$$
d \cong 1.9\left[\frac{\sigma_{L}}{\rho_{L} f^{2}}\right]^{\frac{1}{3}}
$$

where $f$ is the acoustic frequency (varied from $20 \mathrm{kHz}$ to $3 \mathrm{MHz}$ ); $\rho_{\mathrm{L}}$, the liquid density (997 $\mathrm{kg} / \mathrm{m}^{3}$ for water at $25^{\circ} \mathrm{C}$ ); and $\sigma_{\mathrm{L}}$, the liquid surface tension of $72 \mathrm{mN} / \mathrm{m}$. Thus, the mean diameter of the atomized particles was calculated to be $7.9 \mu \mathrm{m}$ for $1 \mathrm{MHz}$ US. The detailed mechanism of atomized particle generation has been reported by Yule and Al-Suleimani ${ }^{(52)}$.

\section{Characteristic radius $r_{0}$ occupied by a single bubble}

In the abovementioned calcein experiment, $110 \mu \mathrm{L}$ of a medium that contained $10 \%$ NBs was irradiated by US. The maximum geometrical characteristic radius $r_{0}$ occupied uniformly by a single NB is given as follows:

$$
r_{0}=\left(\frac{3 A h}{4 \pi N_{0}}\right)^{\frac{1}{3}}
$$

where $A$ is the area of the base of a well in the 48-well plate; $h$, height of the medium; $N_{0}$, the number of US contrast agents. For micelle bubbles, $r_{0}$ was calculated to be $19 \mu \mathrm{m}$ at $N_{0}$ $=3.4 \times 10^{8}$ bubbles $/ \mathrm{mL}$.

\section{References}

(1) H. Zhegn, O. Mukdadi, R. Shandas, Theoretical predictions of harmonic generation from submicron ultrasound contrast agents for nonlinear biomedical ultrasound imaging. Physics in Medicine and Biology, Vol.51, No.3 (2006), pp.557-573.

(2) W. Hayduk, H. Laudie, Prediction of diffusion-coefficients for nonelectrolytes in dilute 
aqueous-solutions. Aiche Journal, Vol.20, No.3 (1974), pp.611-615.

(3) A. Kabalnov, D. Klein, T. Pelura, E. Schutt, J. Weers, Dissolution of multicomponent microbubbles in the bloodstream: 1. Theory. Ultrasound in Medicine and Biology, Vol.24, No.5 (1998), pp.739-749.

(4) E.C. Unger, T. Porter, W. Culp, R. Labell, T. Matsunaga, R. Zutshi, Therapeutic applications of lipid-coated microbubbles. Advanced Drug Delivery Reviews, Vol.56, No.9 (2004), pp.1291-1314.

(5) J.R. Lindner, Microbubbles in medical imaging: current applications and future directions. Nature Reviews Drug Discovery, Vol.3, No.6 (2004), pp.527-532.

(6) R. Bekeredjian, S. Chen, P.A. Frenkel, P.A. Grayburn, R.V. Shohet, Ultrasound-targeted microbubble destruction can repeatedly direct highly specific plasmid expression to the heart. Circulation, Vol.108, No.8 (2003), pp.1022-1026.

(7) T.R. Porter, P.L. Iversen, S. Li, F. Xie, Interaction of diagnostic ultrasound with synthetic oligonucleotide-labeled perfluorocarbon-exposed sonicated dextrose albumin microbubbles. Journal of Ultrasound in Medicine, Vol.15, No.8 (1996), pp.577-584.

(8) S.M. Stieger, C.F. Caskey, R.H. Adamson, S. Qin, F.R. Curry, E.R. Wisner, K.W. Ferrara, Enhancement of vascular permeability with low-frequency contrast-enhanced ultrasound in the chorioallantoic membrane model. Radiology, Vol.243, No.1 (2007), pp.112-121.

(9) A.Y. Ammi, R.O. Cleveland, J. Mamou, G.I. Wang, S.L. Bridal, W.D. O'Brien, Ultrasonic contrast agent shell rupture detected by inertial cavitation and rebound signals. IEEE Transactions on Ultrasonics Ferroelectrics and Frequency Control, Vol.53, No.1 (2006), pp.126-136.

(10) S.H. Chen, X.H. Chen, Y. Wang, K. Kosai, M.J. Finegold, S.S. Rich, S.L. Woo, Combination gene therapy for liver metastasis of colon carcinoma in vivo. The Proceedings of the National Academy of Sciences of the United States of America, Vol.92, No.7 (1995), pp.2577-2581.

(11) P.P. Kamaev, J.D. Hutcheson, M.L. Wilson, M.R. Prausnitz, Quantification of optison bubble size and lifetime during sonication dominant role of secondary cavitation bubbles causing acoustic bioeffects. Journal of the Acoustical Society of America, Vol.115, No.4 (2004), pp.1818-1825.

(12) J. Wu, J. Tong, Experimental study of stability of a contrast agent in an ultrasound field. Ultrasound in Medicine and Biology, Vol.24, No.2 (1998), pp.257-265.

(13) H.R. Guzman, D.X. Nguyen, S. Khan, M.R. Prausnitz, Ultrasound-mediated disruption of cell membranes. I. Quantification of molecular uptake and cell viability. Journal of the Acoustical Society of America, Vol.110, No.1 (2001), pp.588-596.

(14) A. van Wamel, K. Kooiman, M. Harteveld, M. Emmer, F.J. ten Cate, M. Versluis, N. de Jong, Vibrating microbubbles poking individual cells: drug transfer into cells via sonoporation. Journal of Control Release, Vol.112, No.2 (2006), pp.149-155.

(15) T. Li, K. Tachibana, M. Kuroki, Gene transfer with echo-enhanced contrast agents: comparison between Albunex, Optison, and Levovist in mice--initial results. Radiology, Vol.229, No.2 (2003), pp.423-428.

(16) )X. Wang, H.D. Liang, B. Dong, Q.L. Lu, M.J. Blomley, Gene transfer with microbubble ultrasound and plasmid DNA into skeletal muscle of mice: comparison between commercially available microbubble contrast agents. Radiology, Vol.237, No.1 (2005), pp.224-229.

(17) H.R. Guzman, A.J. McNamara, D.X. Nguyen, M.R. Prausnitz, Bioeffects caused by changes in acoustic cavitation bubble density and cell concentration: A unified explanation based on cell-to-bubble ratio and blast radius. Ultrasound in Medicine and Biology, Vol.29, No.8 (2003), pp.1211-1222.

(18) M. Ward, J. Wu, J.F. Chiu, Experimental study of the effects of Optison concentration on sonoporation in vitro. Ultrasound in Medicine and Biology, Vol.26, No.7 (2000), 
pp.1169-1175.

(19) E.N. Harvey, D.K. Barnes, W.D. McElroy, A.H. Whiteley, D.C. Pease, K.W. Cooper, Bubble formation in animals. Journal of cellular and comparative physiology, Vol.24 (1944), pp.1-22.

(20) R.E. Apfel, C.K. Holland, Gauging the likelihood of cavitation from short-pulse, low-duty cycle diagnostic ultrasound. Ultrasound in Medicine and Biology, Vol.17, No.2 (1991), pp.179-185.

(21) W.S. Chen, T.J. Matula, L.A. Crum, The disappearance of ultrasound contrast bubbles: observations of bubble dissolution and cavitation nucleation. Ultrasound in Medicine and Biology, Vol.28, No.6 (2002), pp.793-803.

(22) D.L. Miller, R.M. Thomas, Ultrasound contrast agents nucleate inertial cavitation in vitro. Ultrasound in Medicine and Biology, Vol.21, No.8 (1995), pp.1059-1065.

(23) D.M. Hallow, A.D. Mahajan, T.E. McCutchen, M.R. Prausnitz, Measurement and correlation of acoustic cavitation with cellular bioeffects. Ultrasound in Medicine and Biology, Vol.32, No.7 (2006), pp.1111-1122.

(24) D.M. Skyba, R.J. Price, A.Z. Linka, T.C. Skalak, S. Kaul, Direct in vivo visualization of intravascular destruction of microbubbles by ultrasound and its local effects on tissue. Circulation, Vol.98, No.4 (1998), pp.290-293.

(25) M.J. Shortencarier, P.A. Dayton, S.H. Bloch, P.A. Schumann, T.O. Matsunaga, K.W. Ferrara, A method for radiation-force localized drug delivery using gas-filled lipospheres. IEEE Transactions on Ultrasonics, Ferroelectrics, and Frequency Control, Vol.51, No.7 (2004), pp.822-831.

(26) T. Kodama, Y. Tomita, K. Koshiyama, M.J. Blomley, Transfection effect of microbubbles on cells in superposed ultrasound waves and behavior of cavitation bubble. Ultrasound in Medicine and Biology, Vol.32, No.6 (2006), pp.905-914.

(27) S. Gambihler, M. Delius, J.W. Ellwart, Permeabilization of the plasma-membrane of L1210 mouse leukemia-cells using lithotripter shock-waves. Journal of Membrane Biology, Vol.141, No.3 (1994), pp.267-275.

(28) T. Kodama, A.G. Doukas, M.R. Hamblin, Shock wave-mediated molecular delivery into cells. Biochimica et Biophysica Acta, Vol.1542, No.13 (2002), pp.186-194.

(29) A.G. Doukas, D.J. McAuliffe, T.J. Flotte, Biological effects of laser-induced shock waves: structural and functional cell damage in vitro. Ultrasound in Medicine and Biology, Vol.19, No.2 (1993), pp.137-146.

(30) T. Kodama, M.R. Hamblin, A.G. Doukas, Cytoplasmic molecular delivery with shock waves: importance of impulse. Biophysical Journal, Vol.79, No.4 (2000), pp.1821-1832.

(31) K. Koshiyama, T. Kodama, T. Yano, S. Fujikawa, Structural change in lipid bilayers and water penetration induced by shock waves: molecular dynamics simulations. Biophysical Journal, Vol.91, No.6 (2006), pp.2198-2205.

(32) K. Koshiyama, T. Kodama, T. Yano, S. Fujikawa, Molecular dynamics simulation of structural changes of lipid bilayers induced by shock waves: Effects of incident angles. Biochimica et Biophysica Acta, Vol.1778, No.6 (2008), pp.1423-1428.

(33) K. Koshiyama, T. Kodama, T. Yano, S. Fujikawa, Molecular dynamics simulation of water pore formation in lipid bilayer induced by shock waves. Therapeutic Ultrasound: 5th International Symposium on Therapeutic Ultrasound, Vol. 829, (2005), pp.583-587.

(34) A. Aoi, Y. Watanabe, S. Mori, M. Takahashi, G. Vassaux, T. Kodama, Herpes simplex virus thymidine kinase-mediated suicide gene therapy using nano/microbubbles and ultrasound. Ultrasound in Medicine and Biology, Vol.34, No.3 (2008), pp.425-434.

(35) J.R. Tennant, Evaluation of the trypan blue technique for determination of cell viability. Transplantation, Vol.2 (1964), pp.685-694.

(36) )T. Kodama, A.G. Doukas, M.R. Hamblin, Delivery of ribosome-inactivating protein toxin into cancer cells with shock waves. Cancer Letters, Vol.189, No.1 (2003), pp.69-75. 
(37) M. Postema, A. Bouakaz, M. Versluis, N. de Jong, Ultrasound-induced gas release from contrast agent microbubbles. IEEE Transactions on Ultrasonics, Ferroelectrics, and Frequency Control, Vol.52, No.6 (2005), pp.1035-1041.

(38) B. Wolfrum, R. Mettin, T. Kurz, W. Lauterborn, Observations of pressure-wave-excited contrast agent bubbles in the vicinity of cells. Applied Physics Letters, Vol.81, No.26 (2002), pp.5060-5062.

(39) C.D. Ohl, M. Arora, R. Ikink, N. de Jong, M. Versluis, M. Delius, D. Lohse, Sonoporation from jetting cavitation bubbles. Biophysical Journal, Vol.91, No.11 (2006), pp.4285-4295.

(40) J.R. Blake, B.B. Taib, G. Doherty, Transient cavities near boundaries .1. rigid boundary. Journal of Fluid Mechanics, Vol.170 (1986), pp.479-497.

(41) J.R. Blake, B.B. Taib, G. Doherty, Transient cavities near boundaries .2. free-surface. Journal of Fluid Mechanics, Vol.181 (1987), pp.197-212.

(42) A. Shima, K. Takayama, Y. Tomita, N. Miura, An experimental study on effects of a solid wall on the motion of bubbles and shock waves in bubble collapse. Acustica, Vol.48, No.5 (1981), pp.293-301.

(43) J.B. Keller, M. Miksis, Bubble oscillations of large amplitude. Journal of the Acoustical Society of America, Vol.68, No.2 (1980), pp.628-633.

(44) A. Prosperetti, A. Lezzi, Bubble Dynamics in a Compressible Liquid .1. 1st-Order Theory. Journal of Fluid Mechanics, Vol.168 (1986), pp.457-478.

(45) P.A. Dayton, J.E. Chomas, A.F.H. Lum, J.S. Allen, J.R. Lindner, S.I. Simon, K.W. Ferrara, Optical and acoustical dynamics of microbubble contrast agents inside neutrophils. Biophysical Journal, Vol.80, No.3 (2001), pp.1547-1556.

(46) J. Sundaram, B.R. Mellein, S. Mitragotri, An experimental and theoretical analysis of ultrasound-induced permeabilization of cell membranes. Biophysical Journal, Vol.84, No.5 (2003), pp.3087-3101.

(47) E.A. Evans, R. Waugh, L. Melnik, Elastic area compressibility modulus of red cell membrane. Biophysical Journal, Vol.16, No.6 (1976), pp.585-595.

(48) R. Hickling, M. Plesset, Collapse and rebound of a spherical bubble in water. Physics of Fluids, Vol.7, No.1 (1964), pp.7-14.

(49) R.H. Cole, Underwater Explosions, Princeton University Press, Princeton, NJ, (1948).

(50) Y. Tomita, M. Tsubota, N. An-Naka, Energy evaluation of cavitation bubble generation and shock wave emission by laser focusing in liquid nitrogen. Journal of Applied Physics, Vol.93, No.5 (2003), pp.3039-3048.

(51) B. Alberts, D. Bray, J. Lewis, M. Raff, K. Roberts, J.D. Watson, Molecular biology of the cell, Garland Publishing, Inc., New York \& London, (1994).

(52) A.J. Yule, Y. Al-Suleimani, On droplet formation from capillary waves on a vibrating surface. Proceedings of the Royal Society of London Series a-Mathematical Physical and Engineering Sciences, Vol.456, No.1997 (2000), pp.1069-1085.

(53) S. Bao, B.D. Thrall, D.L. Miller, Transfection of a reporter plasmid into cultured cells by sonoporation in vitro. Ultrasound in Medicine and Biology, Vol.23, No.6 (1997), pp.953-959.

(54) T. Tsujino, A. Shima, The Behavior of Gas-Bubbles in Blood Subjected to an Oscillating Pressure. Journal of Biomechanics, Vol.13, No.5 (1980), pp.407-416.

(55) S. Fujikawa, T. Akamatsu, Effects of the non-equilibrium condensation of vapour on the pressure wave produced by the collapse of a bubble in a liquid. Journal of Fluid Mechanics, Vol.97, No.3 (1980), pp.481-512.

(56) Y. Tomita, A. Shima, On the behavior of a spherical bubble and the impulse pressure in a viscous compressible liquid. Bulletin of the JSME, Vol.20, No.149 (1977), pp.1453-1460.

(57) C. Chiba, Study on atomization of liquid due to ultrasound oscillation. Ph.D. Thesis, Engineering, Tohoku University, Sendai, (1983). 